\title{
Analysis of adiabatic heating and its influence on the Garofalo equation parameters of a high nitrogen steel
}

\author{
J. Castellanos ${ }^{\text {a }}$ I. Rieiro ${ }^{\text {a }}$, M. Carsí ${ }^{\text {b }}$ J. Muñoz ${ }^{a}$, M. El Mehtedic, O.A. Ruano ${ }^{\text {b, }}$ \\ ${ }^{a}$ Dept. of Mathematics, Universidad de Castilla-La Mancha, Av. Carlos III, s/n, E- \\ 45004 Toledo (Spain) \\ ${ }^{\mathrm{b}}$ Dept. of Physical Metallurgy, Centro Nacional de Investigaciones Metalúrgicas, \\ C.S.I.C., Av. Gregorio del Amo 8, E-28040, Madrid, Spain \\ ${ }^{\mathrm{c}}$ Dept. of Mechanics, Polytechnic University of Marche, Via Brecce Bianche, I-60131 \\ Ancona, Italy \\ * Corresponding author: Tel. +34 91 5538900; fax: +34 915347425 \\ E-mail address: ruano@cenim.csic.es (O.A. Ruano)
}

\begin{abstract}
.
Torsion tests at high temperatures and high strain rates were conducted on a high nitrogen steel (HNS). Under these conditions, adiabatic heating influences its flow behavior. This work focus on a new algorithm for conducting the adiabatic heating correction of stress-strain curves. The algorithm obtains the stress-strain curves at quasiisothermal conditions from those at adiabatic conditions. The corrections in stress obtained can be higher than $15 \%$ and increase with increasing strain rates and decreasing temperatures. On the other hand, an upper bound for the temperature rise was found using a dynamic material behavior approach. Finally, the influence of adiabatic heating correction on the Garofalo equation parameters of HNS was analyzed. High values of activation energy and stress exponent were attributed to reinforcement by dispersed carbonitrides and the high amount of alloying elements.
\end{abstract}

Keywords: Torsion tests; Adiabatic heating; Garofalo equation; High nitrogen steel; Modelling 


\section{Introduction}

Torsion tests at high temperature and moderate and high strain rate usually lead to a temperature rise of the specimen with deformation, called adiabatic heating. In this situation, the stress-strain curves obtained are not at constant temperature [1]. Under adiabatic conditions, the stresses are lower than those under isothermal conditions. Therefore the approximation of adiabatic stress-strain curves (or torque-number of turns curves) by the isothermal ones could lead to some errors in various quantitative analysis of the material creep behavior. For instance, the determination of the stress from the torque is usually carried out by the Fields-Backofen equation [2]:

$\sigma=\frac{\sqrt{3} \Gamma}{2 \pi R^{3}}\left(3+n^{\prime}+m^{\prime}\right)$

where $\sigma$ is the stress, $\Gamma$ is the torque, $R$ is the radius of the torsion sample, and n' and $\mathrm{m}$ ' are the work hardening and rate sensitivity of the torque respectively, which are defined as follow:

$$
n^{\prime}=\left.\frac{\partial \ln \Gamma}{\partial \ln N}\right|_{\dot{N}, T} \quad m^{\prime}=\left.\frac{\partial \ln \Gamma}{\partial \ln \dot{N}}\right|_{N, T}
$$

where $\mathrm{N}$ is the number of turns and $\dot{N}$ is the speed rotation.

The determination of the stress by Eq. (1) assumes isothermal conditions for deformation through the Eqs. (2) and (3). However, these equations should be different under adiabatic conditions which would lead to errors in the determination of the stress.

Similarly, the Garofalo equation as a function of strain is usually employed for the fitting of the stress, strain rate and temperature data, $\sigma, \dot{\varepsilon}$ and T, respectively [3-5]. This equation is especially adequate for correlation of creep data in wide ranges of temperature and strain rate [6]. The Garofalo equation is given by: 
$\dot{\varepsilon}=A \exp \left(-\frac{Q}{R T}\right) \sinh ^{n}(\alpha \sigma)$

where $\mathrm{Q}$ is the activation energy for deformation, $\mathrm{n}$ is the stress exponent, $\mathrm{A}$ is the preexponential factor, $\alpha$ is the stress co-factor and $\mathrm{R}$ is the gas constant. The Garofalo equation parameters are sensible to the values of stress and temperature which are affected by adiabatic heating of the sample. Again, the approximation of adiabatic stress-strain curves by the isothermal ones could lead to some errors in the determination of such parameters.

Finally, to carry out dynamic recrystallization (DRX) studies through the Avrami equation $[7,8]$ without consideration of the effects of adiabatic heating could lead to errors in the determination of the softening behavior of DRX since part of this softening is related to adiabatic heating.

All the previous examples show the importance of obtaining stress-strain curves at isothermal conditions from data obtained at adiabatic conditions. Therefore, the aims of this work are a) to propose a new iterative algorithm to carry out the adiabatic heating correction of stress-strain curves and b) to study the influence of the adiabatic heating correction on the Garofalo equation parameters of a high nitrogen steel (HNS).

Moreover, the adiabatic heating correction proposed is complemented by a determination of an upper bound for the temperature rise of the bulk material following the hypothesis of Prasad which considers the sample as a power dissipator [9].

\section{Theoretical approach}

\subsection{Determination of the stress at isothermal conditions.}


A new iterative algorithm to carry out the adiabatic heating correction is presented. The algorithm is based on two equations. The first one is the usual equation for the temperature rise with strain of the bulk material during deformation, $\Delta \mathrm{T}[1,10,11]$ :

$$
\Delta T=\frac{\eta}{\rho C} \int_{0}^{\varepsilon} \sigma_{0}^{w c}(s) d s
$$

where $\eta$ is the Taylor-Quinney factor, $\rho$ is the density, $\mathrm{C}$ is the specific heat and $\sigma_{0}{ }^{\text {wc }}$ is the stress without adiabatic heating correction (given by Eq. (1)).

The second equation allows the calculation of the stresses under isothermal conditions $\sigma_{0}{ }^{\mathrm{c}}$, from the ones under adiabatic conditions $\sigma_{0}{ }^{\mathrm{wc}}$. For a given test at constant $\dot{\varepsilon}$ and initial temperature T, and for a given value of $\varepsilon$, the uncorrected stress $\sigma_{0}{ }^{\text {wc }}$ (associated with adiabatic conditions), can be expressed as a function of the corrected stress, $\sigma_{0}{ }^{\mathrm{c}}$ (associated with isothermal conditions) as:

$\sigma_{0}^{w c}(\varepsilon, \dot{\varepsilon}, T-\Delta T)=\sigma_{0}^{c}(\varepsilon, \dot{\varepsilon}, T)$

Applying a first order Taylor expansion of the function $\sigma_{0}{ }^{\mathrm{wc}}$ in Eq. (6) about the point $\mathrm{T}$ it is obtained:

$$
\sigma_{0}^{c}(\varepsilon, \dot{\varepsilon}, T)=\sigma_{0}^{w c}(\varepsilon, \dot{\varepsilon}, T)-\left.\frac{\partial \sigma_{0}^{w c}(T)}{\partial T}\right|_{\varepsilon, \dot{\varepsilon}} \Delta T
$$

An expression, similar to Eq. (7), was recently used by Kapoor et al. [12]. It should be noted that the application of Eqs. (5) and (7) lead to errors in the determination of $\Delta \mathrm{T}$ and $\sigma_{0}{ }^{\mathrm{c}}$ because isothermal conditions are assumed for the calculation of the uncorrected stress in Eqs. (1) to (3) whereas Eqs. (5) and (7) assumes adiabatic conditions for the same stress. A new iterative algorithm is introduced in the next section to correct this problem. 


\subsection{Description and justification of the iterative algorithm for the adiabatic heating} correction

The application of Eq. (5) for the temperature rise of the bulk material during torsion testing can lead to some errors since the stress $\sigma_{0}{ }^{\mathrm{wc}}$ is obtained assuming isothermal conditions for deformation (Eqs. (1) to (3)). In other words, the calculation of n' and m' is done assuming isothermal conditions which is not true since the experimental curves are obtained under adiabatic conditions.

On the other hand, the determination of the corrected stress given in Eq. (7) is strongly influenced by the partial derivative of the uncorrected stress with respect to the temperature. In turn, the values of this partial derivative are strongly influenced by adiabatic heating. Fig. 1 shows, in fine lines, the isothermal stress-strain curves for a given strain rate $\dot{\varepsilon}$ and two initial temperatures $\mathrm{T}_{0}{ }^{1}$ and $\mathrm{T}_{0}{ }^{2}$, and in thick lines, the corresponding curves without adiabatic correction. The isothermal curves are contained in a two dimensional space $\{\varepsilon, \sigma\}$ while the adiabatic curves are in a three dimensional space $\{\varepsilon, \sigma, T\}$.

According to Fig. 1, for a given strain $\varepsilon_{0}$ the partial derivative can be calculated, in a first approximation, as:

$\frac{\partial \sigma_{0}^{w c}(T)}{\partial T}=\frac{\sigma^{(2)}-\sigma^{(1)}}{T_{0}^{2}-T_{0}^{1}}$

This expression is subjected to errors due because the partial derivative is affected by the different temperature increments at $\varepsilon_{0}$ of the two adiabatic curves $\Delta \mathrm{T}^{(1)}$ and $\Delta \mathrm{T}^{(2)}$. Considering the effect of the temperature increase, Eq. (8) is transformed as follows:

$$
\frac{\partial \sigma_{0}^{w c}(T)}{\partial T}=\frac{\sigma^{(2)}-\sigma^{(1)}}{\left(T_{0}^{(2)}+\Delta T^{(2)}\right)-\left(T_{0}^{(1)}+\Delta T^{(1)}\right)}
$$


This equation is more accurate than Eq. (8), but still has some level of inaccuracy since the stress in Eq. (9) was calculated assuming isothermal conditions. In addition, the two temperature increments, $\Delta \mathrm{T}^{(1)}$ and $\Delta \mathrm{T}^{(2)}$, have errors since they were calculated through Eq. (5).

In order to solve the problems mentioned previously an iterative algorithm for the adiabatic heating correction has been developed. This algorithm, fully described in Appendix A, starts with an initial estimation of the temperature rise and the corrected stress by means of Eq. (7). The increment of temperature that is added in the iteration $\mathrm{k}$ to the initial estimation is calculated from the difference between the corrected and uncorrected stress in the iteration $\mathrm{k}-1$. On the other hand, the corrected stress that is added in the iteration $\mathrm{k}$ to the initial estimation is calculated applying Eq. (7) with the temperature rise obtained in iteration $\mathrm{k}$. The correction has been carried out starting at the peak strain since previous to this value, in the first part of deformation, adiabatic heating has not an important effect on the stress $[13,14]$ and therefore the adiabatic heating correction may distort the values of stress close to the peak affecting further studies such as determination of a constitutive relation or analysis of DRX.

\subsection{Upper bound for the temperature rise}

An upper bound for the temperature rise is proposed in order to validate the results given for the adiabatic heating correction stated in the previous section.

For a given $\mathrm{T}$ and $\dot{\varepsilon}$, the power $\mathrm{P}$ (per unit volume) absorbed by the material during plastic flow is given by:

$$
\begin{aligned}
& \sigma \dot{\varepsilon}=\int_{0}^{\dot{\varepsilon}} \sigma d \dot{\varepsilon}+\int_{0}^{\sigma} \dot{\varepsilon} d \sigma \\
& P=G+J
\end{aligned}
$$


where $\mathrm{G}$ and $\mathrm{J}$ are the dissipator content and co-content respectively. The $\mathrm{G}$ term represents the power dissipated to plastic work, most of which is converted into heat [9].

On the other hand, the Garofalo equation can be expressed as follows:

$$
\sigma(\dot{\varepsilon})=\frac{1}{\alpha} \sinh ^{-1}\left[\left(Z_{A}\right)^{\frac{1}{n}}\right]
$$

where $\mathrm{Z}_{\mathrm{A}}$ is the Zener-Hollomon parameter compensated with $\mathrm{A}$ (pre-exponential constant of Garofalo equation): $Z_{A}=\dot{\varepsilon} / A \exp (Q / R T)$. Substituting Eq. (12) in the expression for $\mathrm{G}$ given by Eqs. (10) and (11) and considering that $\sinh ^{-1}(x) \leq x$ the following expression is obtained:

$$
G(\dot{\varepsilon})=\int_{0}^{\dot{\varepsilon}} \sigma d \dot{\varepsilon}=\int_{0}^{\dot{\varepsilon}} \frac{1}{\alpha} \sinh ^{-1}\left(\left(Z_{A}\right)^{\frac{1}{n}}\right) \leq \int_{0}^{\dot{\varepsilon}} \frac{1}{\alpha}\left(Z_{A}\right)^{\frac{1}{n}}=\frac{n}{\alpha(n+1)} \dot{\varepsilon} \sinh \left(\alpha \sigma_{p}\right)=\bar{G}(\dot{\varepsilon})
$$

where $\sigma_{\mathrm{p}}$ is the peak stress. $\bar{G}(\dot{\varepsilon})$ represents an upper bound for the energy available to be converted into heat and in consequence to increase the temperature of the bulk material. Using Eqs. (5) and (13), the following upper bound for the temperature rise is obtained:

$$
\Delta \bar{T}=\frac{n \varepsilon}{\alpha(n+1)} \sinh \left(\alpha \sigma_{p}\right)
$$

where the Taylor-Quinney factor is taken as 1 for simplification. As expected, $\Delta \bar{T}$ increases with increasing strain and increasing peak stress.

\section{Material and experimental method}


The chemical composition of the HNS, Cronidur ${ }^{\circledR} 30$, is the following (wt.\%): $0.34 \mathrm{C}$ $0.33 \mathrm{~N}-0.45 \mathrm{Mn}-16.2 \mathrm{Cr}-1.1 \mathrm{Mo}$. This is a martensitic through-hardened steel that can also be induction case-hardened. The steel is characterized by a homogeneous structure of finely dispersed carbonitrides.

The torsion tests were carried out on the torsion machine placed at the laboratory of the Department of Mechanics (Polytechnic University of Marche), at temperatures varying in the range $900-1200^{\circ} \mathrm{C}$, and equivalent strain rates from 0.005 to $5 \mathrm{~s}^{-1}$, up to rupture, in controlled Argon atmosphere to avoid oxidation and minimize the effects of adiabatic heating.

The torsion samples were machined from billets with the axis parallel to the cast direction. The gauge section of the samples was a solid cylinder with a length of 15 $\mathrm{mm}$, and a radius of $5 \mathrm{~mm}$. The samples were heated by a high frequency induction coil $\left(1{ }^{\circ} \mathrm{C} / \mathrm{s}\right)$ up to $1200^{\circ} \mathrm{C}$, hold $10 \mathrm{~min}$ at temperature, cooled to the deformation temperature at $1{ }^{\circ} \mathrm{C} / \mathrm{s}$ and hold at deformation temperature for other $5 \mathrm{~min}$ before testing. The experimental method is described in detail elsewhere ${ }^{10)}$.

The specific heat of the HNS is considered $800 \mathrm{JKg}^{-1} \cdot \mathrm{k}^{-1}$ for all the temperature range and the density is varying with temperature according to the relation: $\rho\left(\mathrm{kg} \cdot \mathrm{m}^{-3}\right)=-$ $0.4 * \mathrm{~T}\left({ }^{\circ} \mathrm{C}\right)+7925.9$ [15]. Finally, the Taylor-Quinney coefficient is taken equal to 0.8 .

\section{Results and discussion}

\subsection{Analysis of adiabatic heating on the stress-strain curves}

Fig. 2 shows stress-strain curves for all the strain rates and initial temperatures of the torsion tests. The thick lines represent the curves at isothermal conditions (corrected stress), calculated by the application of the algorithm previously presented, and the fine lines are the ones for adiabatic conditions (uncorrected stress). As mentioned before, the 
correction was carried out from the peak to a value of the strain where it is considered that the effect of flow localization is not important. At temperatures higher than $900^{\circ} \mathrm{C}$ the curves show a clearer steady state under isothermal conditions than under adiabatic conditions. The adiabatic heating increases with increasing strain rate and strain and decreasing temperature. This is because there is more mechanical energy available to be converted into heat under these conditions.

Except for $900^{\circ} \mathrm{C}$, the flow behavior of the material under adiabatic and isothermal conditions is described by a continuous increase of the stress to a peak value followed by a decrease to a steady-state previous to rupture. It is generally accepted that this behavior is characterized by intense work hardening prior to dynamic recovery which is followed by dynamic recrystallization as the two main softening processes. On the other hand, dynamic recrystallization is not present at $900^{\circ} \mathrm{C}$. The low ductility at this temperature has been attributed to the large grain size of the primary austenite [16].

Table 1 shows the absolute increment of stress given by the adiabatic heating correction at a) $\varepsilon=1.5$ and b) $\varepsilon=3$ and various forming conditions. It can be seen that the stress difference increases with increasing strain rate and strain and decreasing temperature. At $\varepsilon=1.5$ the increment of stress is about $7 \%$ while at $\varepsilon=3$ it can be higher than $15 \%$. These results show that the adiabatic heating correction conducts to significant differences in stress between the adiabatic and isothermal conditions. Therefore, the effects of adiabatic heating must be considered for its influence on the Garofalo equation parameters which are necessary for any constitutive modelling.

Figure 3 shows the calculated temperature rise at a) $0.5 \mathrm{~s}^{-1}$ and b) $5 \mathrm{~s}^{-1}$ and various initial temperatures given by the iterative algorithm. A quasi-linear behavior for the temperature rise as a function of strain is observed. Similar linear temperature rise has been previously reported at low and warm temperatures and very high strain rates for 
other steels $[17,18]$. For $\varepsilon=3$ the temperature rise varies from the minimum value of $12.5^{\circ} \mathrm{C}$ at $0.005 \mathrm{~s}^{-1}$ and $1200{ }^{\circ} \mathrm{C}$ to the maximum value of $65^{\circ} \mathrm{C}$ at $5 \mathrm{~s}^{-1}$ and $1050^{\circ} \mathrm{C}$.

In addition, Fig. 4 shows the temperature rise and its upper bound at $0.5 \mathrm{~s}^{-1}$ and different initial temperatures. The thick line represents the upper bound for the temperature rise given by Eq. (14) and the fine line show the temperature rise obtained by the application of the iterative algorithm for the adiabatic heating correction. As expected, the upper bound is always above the calculated temperature rise.

A comparison of two methods for adiabatic heating correction is given in Fig. 5. The first is the traditional method where the stress is corrected through Eqs. (5) and (7). This corresponds in the figure to the two lower curves. The other method is that proposed in this work by means of the described iterative algorithm. It should be noted that the uncorrected curves are not the same since in the traditional method the uncorrected stress was calculated assuming n' $=0$ and $m^{\prime}=0.23$, Eqs. (2) and (3), while the uncorrected stress in the proposed method is determined using the same equations but considering n' and m' as functions of strain rate, strain and temperature. A plateau in the stress- strain curve under isothermal conditions using the proposed method is observed. This suggests that a steady state may be present in the torsion curves even when a strong decrease in stress with strain is observed under uncorrected conditions.

\subsection{Influence of the adiabatic heating correction on the parameters of the Garofalo equation as a function of the strain.}

Figure 6 shows the evolution of the Garofalo parameters for the HNS steel as a function of strain. The Garofalo equation parameters were determined by means of an algorithm specifically designed for the fit of this equation [4]. The fits were carried out at strain values of $0.5,1,1.5,2,2.5$ and 3 . The curves reveal that a steady state is more 
clearly defined at strains higher than 2 in the case of adiabatic heating correction. In addition, it is observed that the adiabatic heating correction is not significant for strains lower than 1. Except for the stress exponent, $n$, the values of $Q, \alpha$ and $\ln (\mathrm{A})$ are significantly affected by the adiabatic heating correction at large strains in this steel. On the other hand, the activation energy obtained at the steady state is too high to be related to the self-diffusivity of iron in the austenite, $270 \mathrm{~kJ} \mathrm{~mol}^{-1}$, but it is similar to that obtained for other previously reported steels [3, 19]. It is attributed to dispersed carbonitrides and the high amount of alloying elements of the steel. Finally, the value of $\mathrm{n}$ is higher than that given by the slip creep equation, 5, corresponding to a power law equation [20]. It should be noted that the $\mathrm{n}$ parameter of the Garofalo equation is different from that of the power law equation since it is affected by the $\alpha$ value [21]. Again, the high $\mathrm{n}$ value is attributed to the high amount of particles that makes the steel to behave as a reinforced material.

Table 2 shows the Garofalo equation parameters as a function of strain. The parameters are obtained from the isothermal curves. Columns 6 and 7 indicate the goodness of the fittings obtained through the $\mathrm{R}^{2}$ and $\mathrm{F}$ of Fisher-Snedecor parameters. The high values of these parameters ensure that the Garofalo equation is capable of correlating, at all strains, the stress data in the wide ranges of the strain rate and temperature considered.

\section{Conclusions}

1. The new proposed algorithm for the adiabatic heating correction is an improvement over the traditional method since the inaccuracies associated to the latter are minimized. 
2. The adiabatic heating correction leads to difference in stress higher than $15 \%$ showing the relevance of adiabatic heating especially at high strains.

3. The correction applied to the high nitrogen steel leads to a clear steady state regime between 2 and 4 in the stress-strain curves.

4. The Garofalo equation parameters are strongly affected by adiabatic heating showing quite stable values for strains in the range of 1 to 3 . The high values of activation energy and stress exponent are attributed to reinforcement by dispersed carbonitrides and the high amount of alloying elements in this steel.

\section{Acknowledgements}

The work was carried out through the Projects PBC-05-010-1 from JCCM (CastillaLa Mancha, Spain) and MAT2006-13348 from CICYT. Thanks are given to Prof. E Evangelista for valuable assistance.

\section{Appendix A}

The initial estimations for the temperature rise and the corrected stress $\Delta \mathrm{T}_{0}$ and $\sigma_{0}{ }^{\mathrm{c}}$ respectively are given by the equations:

$$
\begin{aligned}
& \Delta T_{0}\left(\varepsilon_{i}\right)=\frac{\eta}{\rho C} \int_{\varepsilon_{p}}^{\varepsilon_{i}} \sigma_{0}^{w c}\left(\varepsilon_{i}, \dot{\varepsilon}_{j}, T\right) d \varepsilon \\
& \sigma_{0}^{c}\left(\varepsilon_{i}, \dot{\varepsilon}_{j}, T\right)=\sigma_{0}^{w c}\left(\varepsilon_{i}, \dot{\varepsilon}_{j}, T\right)-\frac{\partial \sigma_{0}^{w c}\left(\varepsilon_{i}, \dot{\varepsilon}_{j}, T\right)}{\partial T} \frac{\eta}{\rho C} \int_{\varepsilon_{p}}^{\varepsilon_{i}} \sigma_{0}^{w c}\left(\varepsilon_{i}, \dot{\varepsilon}_{j}, T\right) d \varepsilon
\end{aligned}
$$

where $\varepsilon_{\mathrm{p}}$ is the peak strain.

The iterative algorithm is a modular process in three steps. In the first, the value of $\Delta \mathrm{T}_{\mathrm{k}}\left(\varepsilon_{\mathrm{i}}\right)$ which is added to the initial estimation of the temperature rise in the iteration $\mathrm{k}$ 
(from $\mathrm{k}=1$ to the number of iterations) is calculated according to the following expression:

$$
\Delta T_{k}\left(\varepsilon_{i}\right)=\frac{\eta}{\rho C} \int_{\varepsilon_{p}}^{\varepsilon_{i}}\left(\sigma_{k-1}^{c}-\sigma_{k-1}^{w c}\right) d \varepsilon
$$

where $\sigma_{\mathrm{k}-1}{ }^{\mathrm{c}}$ and $\sigma_{\mathrm{k}-1}{ }^{\mathrm{wc}}$ are the corrected and the uncorrected stress in the k-1 iteration respectively. In the second module, the partial derivative with respect to $\mathrm{T}$ of the uncorrected stress in the iteration $\mathrm{k}$, is calculated by means of the expression:

$$
\frac{\partial \sigma_{k}^{w c}\left(T_{s}, \varepsilon_{i}\right)}{\partial T} \approx \frac{\sigma_{k}^{w c}\left(T_{s+1}, \varepsilon_{i}\right)-\sigma_{k}^{w c}\left(T_{s}, \varepsilon_{i}\right)}{T_{s+1}-T_{s}}
$$

where $s+1$ refers to the test conducted at the same strain rate but at temperature $T_{k+1}$, that is next in the ascendant sequence. Finally, in the third module, the value of the corrected stress which is added to the initial estimation in the iteration $\mathrm{k}$ is given as:

$\sigma_{k}^{c}\left(\varepsilon_{i}, \dot{\varepsilon}_{j}, T\right)=\sigma_{k}^{w c}\left(\varepsilon_{i}, \dot{\varepsilon}_{j}, T\right)-\frac{\partial \sigma_{0}^{w c}\left(\varepsilon_{i}, \dot{\varepsilon}_{j}, T\right)}{\partial T} \Delta T_{k}\left(\varepsilon_{i}\right)$

The criterion adopted for stopping the algorithm, i.e. the termination tolerance, is $\Delta \sigma \leq 10^{-2} \mathrm{MPa}$ for a given control strain.

\section{References}

[1] W. Pantleon, D. Francke, P. Klimanek, Comput. Mater. Sci. 7 (1996) 75-81.

[2] D.S. Fields, W.A. Backofen, Proc. $60^{\text {th }}$ Annual Meeting of the American Society for Testing Materials, Philadelphia, (1957) 1259.

[3] H.J. McQueen, N.D. Ryan, Mater. Sci. Eng. A 322 (2002) 43-63. 
[4] I. Rieiro, M. Carsí, O.A. Ruano, Mater. Sci. Technol. (2008), doi:

10.1179/174328408X369429.

[5] Y.C. Lin, M.S. Chen, J. Zhang, Mater. Sci. Eng. A (2008), doi: 10.1016/j.msea. 2007.11.119.

[6] I. Rieiro, O.A. Ruano, M. Eddahbi, M. Carsí , J. Mater. Process. Technol. 78 (1998) 177-183.

[7] C.A.C. Imbert, H.J. McQueen, Mater. Sci. Eng. A 313 (2001) 104-116.

[8] C.M. Sellars, W.J. McTegart, Acta Metall. 14, (1966) 1136-1138.

[9] Y.V.R.K. Prasad, H.L. Gegel, S.M. Doraivelu, J.C. Malas, J.T. Morgan, K. A. Lark, D.R. Barker, Metall. Trans. A 15 (1984) 1883-1892.

[10] R. Kapoor, S. Nemat-Nasser, Mech. Mater. 27 (1998) 1-12.

[11] L. Li, J. Zhou, J. Duszczyk, J. Mater. Process. Technol. 172 (2006) 372-380.

[12] R. Kapoor, J.B. Singh, J.K. Chakravartty, Mater. Sci. Eng. A 496 (2008) 308-315.

[13] D. Rittel, Z.G. Wang, Mech. Mater. 40 (2008) 629-635.

[14] Y. Liu, R. Hu, J. Li, H. Kou, H. Li, H. Chang, H. Fu, Mater. Sci. Eng. A 497 (2008) 283-289.

[15] M. F. Rothman, High Temperature Property Data: Ferrous Alloys; ASM International, (1988) pp. 9.9.

[16] M. El Mehtedi, PhD thesis, Università Politecnica delle Marche, Ancona, Italy, 2005 .

[17] Z. Xu, Y. Li, Mech. Mater. (2008), doi: 10.1016/j.mechmat.2008.10.005.

[18] W.S. Lee, C.Y. Liu, Mater. Sci. Eng. A 426 (2006) 101-113.

[19] J.A. DeAlmeida, R. Barbosa, ISIJ Int. 45 (2005) 296

[20] M. Carsí, A. Fernández-Vicente, O.A. Ruano, O.D. Sherby, Mater. Sci. Technol. 15 (1999) 1087 
[21] I. Rieiro, J. Castellanos, J. Muñoz, F. Peñalba, M. Carsí, O. A. Ruano, Proc. $10^{\text {th }}$ Congreso de la Sociedad Española de Materiales, Donostia, 2008. 


\section{Figure captions}

Fig. 1. Stress-strain curves for isothermal and adiabatic conditions, in fine and thick lines, respectively.

Fig. 2. Stress-strain curves for the high nitrogen steel (HNS) at various initial temperatures and at a) $0.005 \mathrm{~s}^{-1}$, b) $0.05 \mathrm{~s}^{-1}$, c) $0.5 \mathrm{~s}^{-1}$ and d) $5 \mathrm{~s}^{-1}$. The thick lines are the isothermal curves (corrected for adiabatic heating) and the fine lines are the adiabatic ones (uncorrected curves).

Fig. 3. Calculated temperature rise with strain at a) $0.5 \mathrm{~s}^{-1}$ and b) $5 \mathrm{~s}^{-1}$ and various initial temperatures $\left(\mathrm{T}_{0}\right)$.

Fig. 4. Upper bounds for the temperature rise, represented by thick lines, Eq. (14), and temperature rise calculated by the iterative algorithm for the adiabatic heating correction, represented by fine lines, as a function of strain at $0.5 \mathrm{~s}^{-1}$ and various initial temperatures.

Fig. 5. A comparison of two methods for the adiabatic heating correction at $5 \mathrm{~s}^{-1}$ and $1200{ }^{\circ} \mathrm{C}$.

Fig. 6. Evolution of the Garofalo equation parameters with strain. The open circles are the parameters obtained from data corrected for adiabatic heating and the filled circles are uncorrected data. 


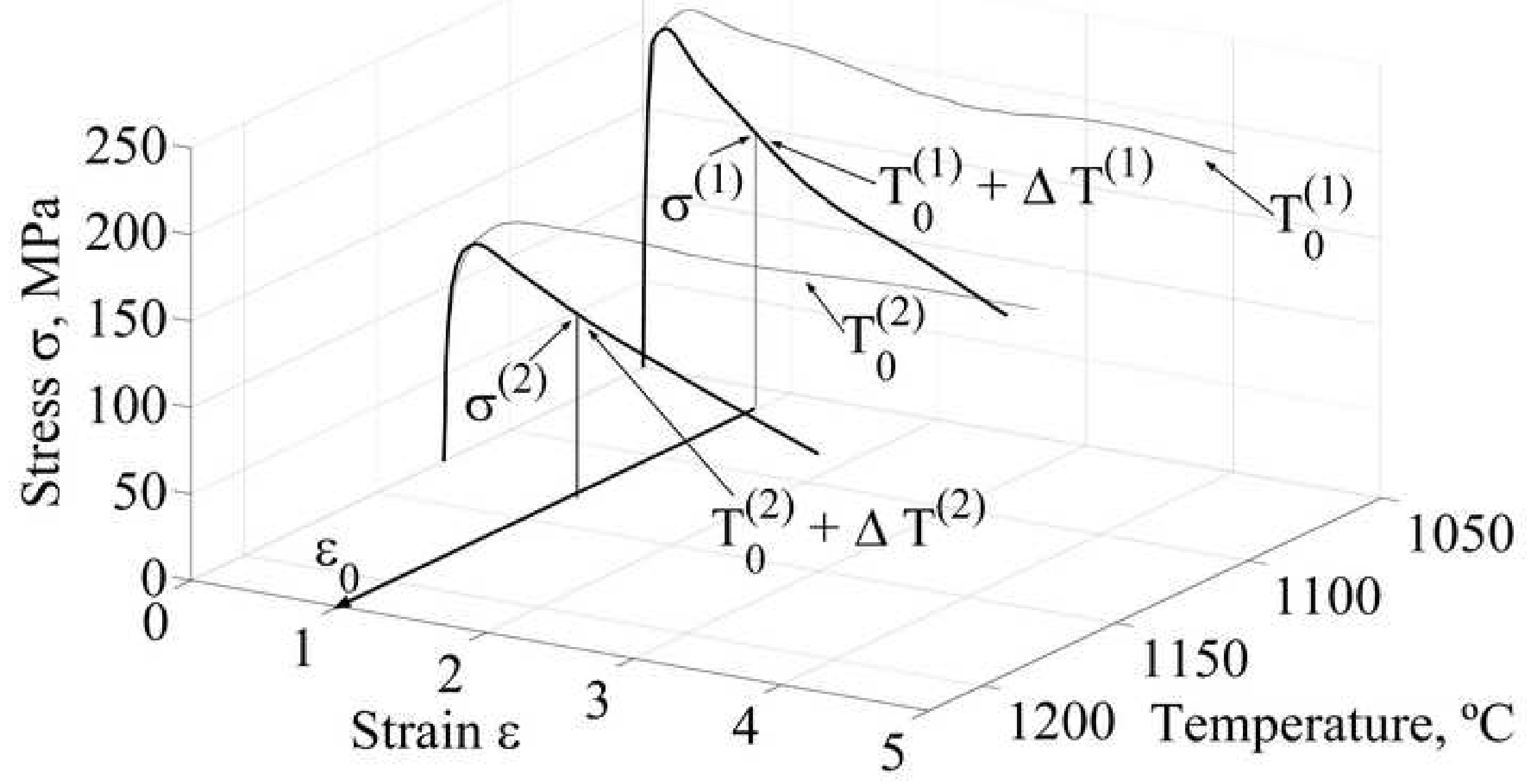



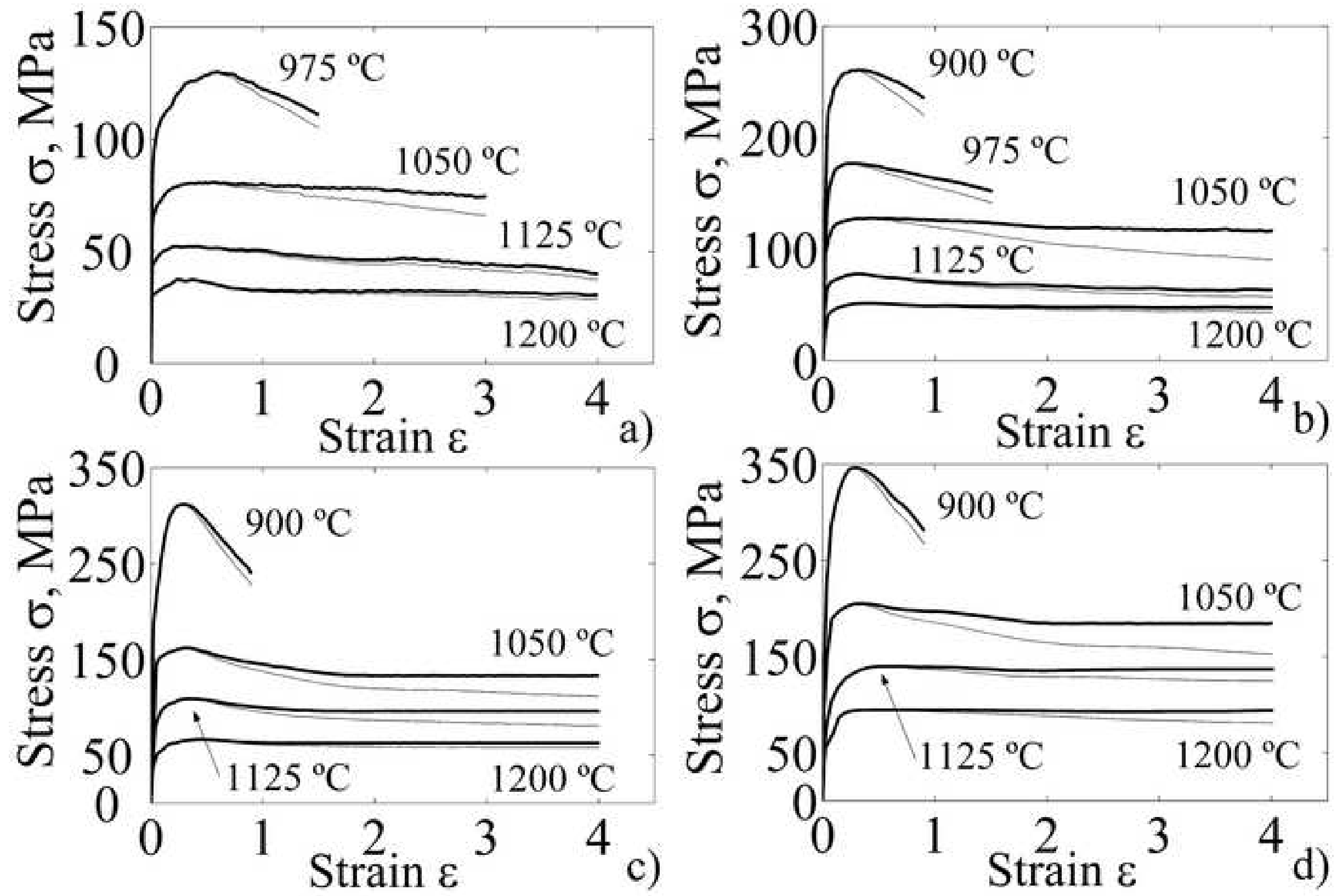

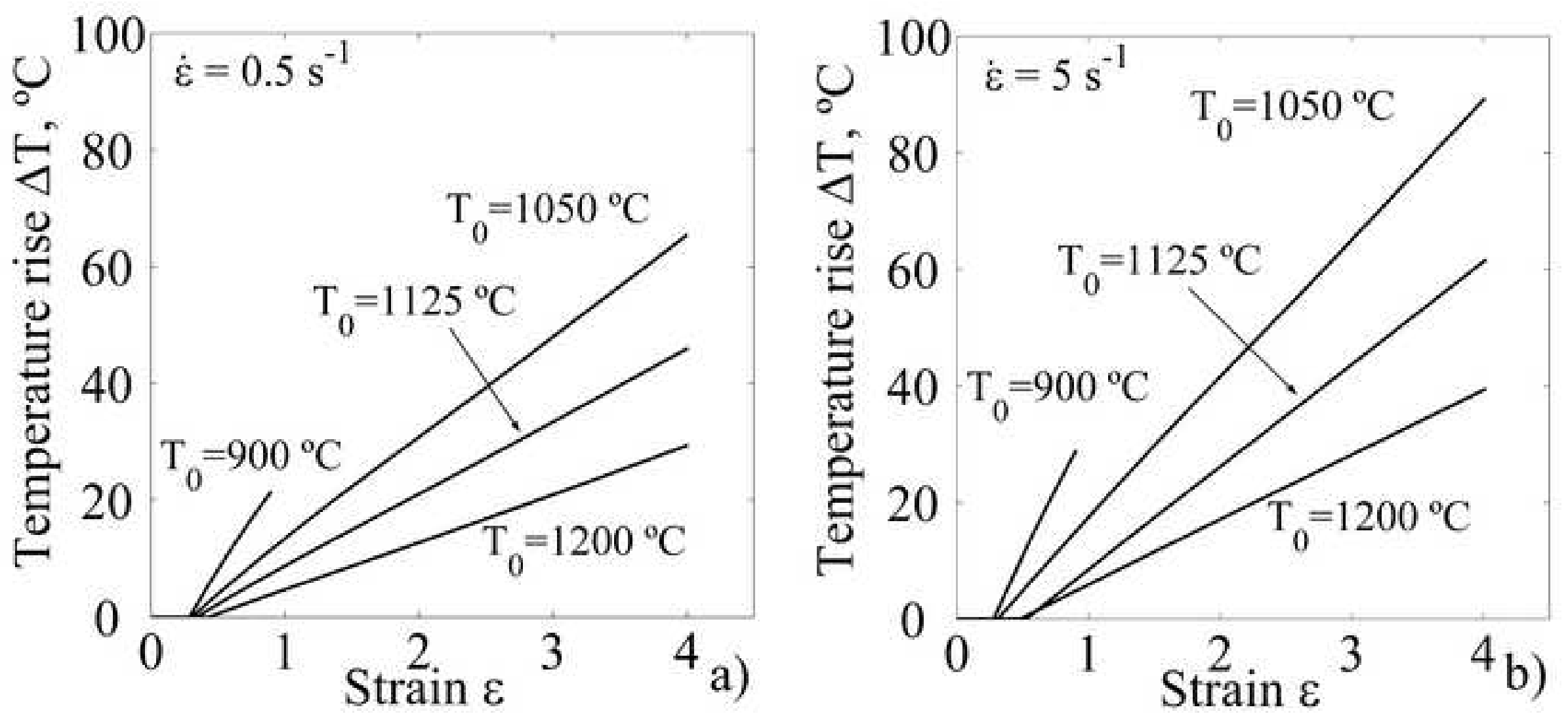

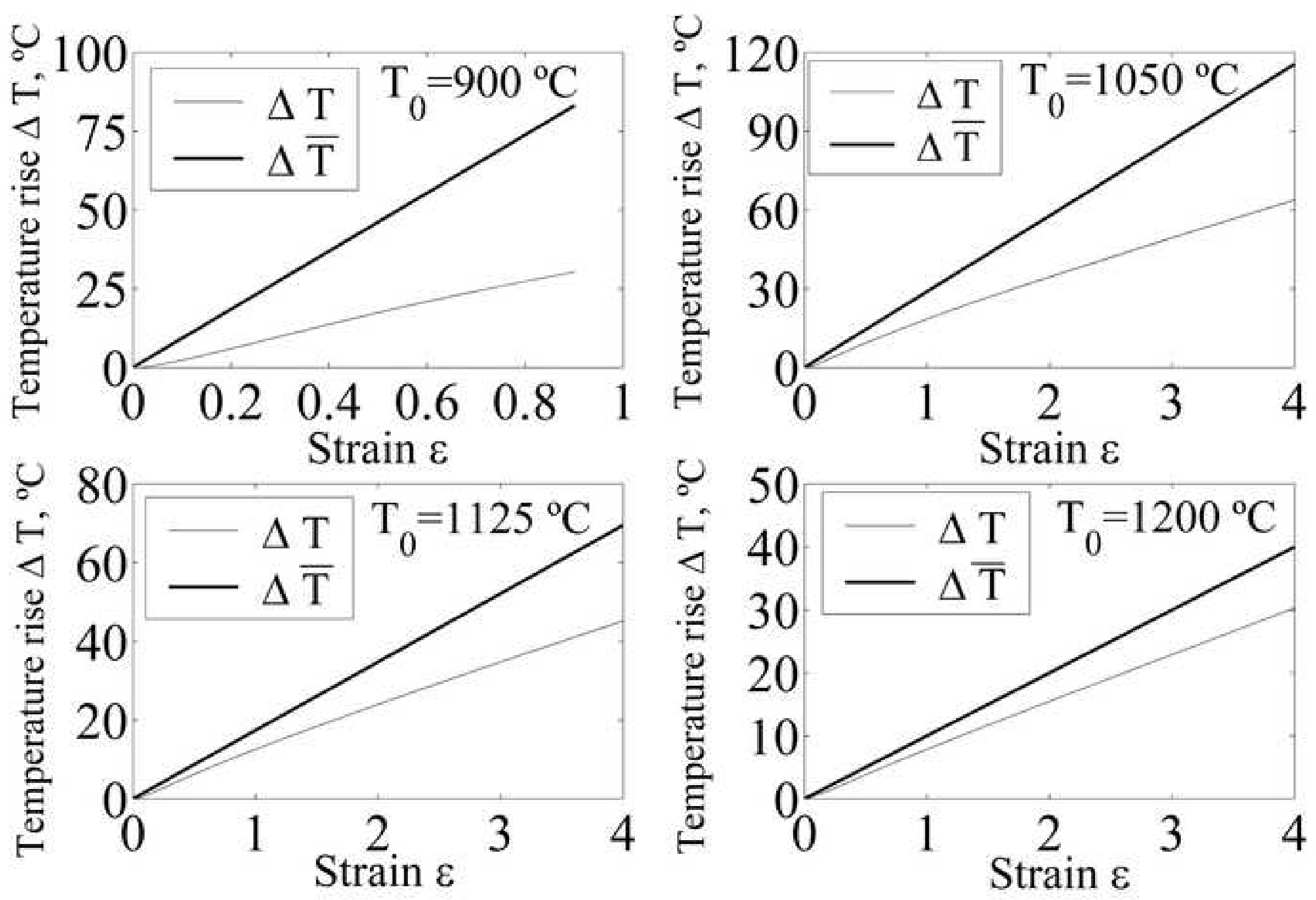


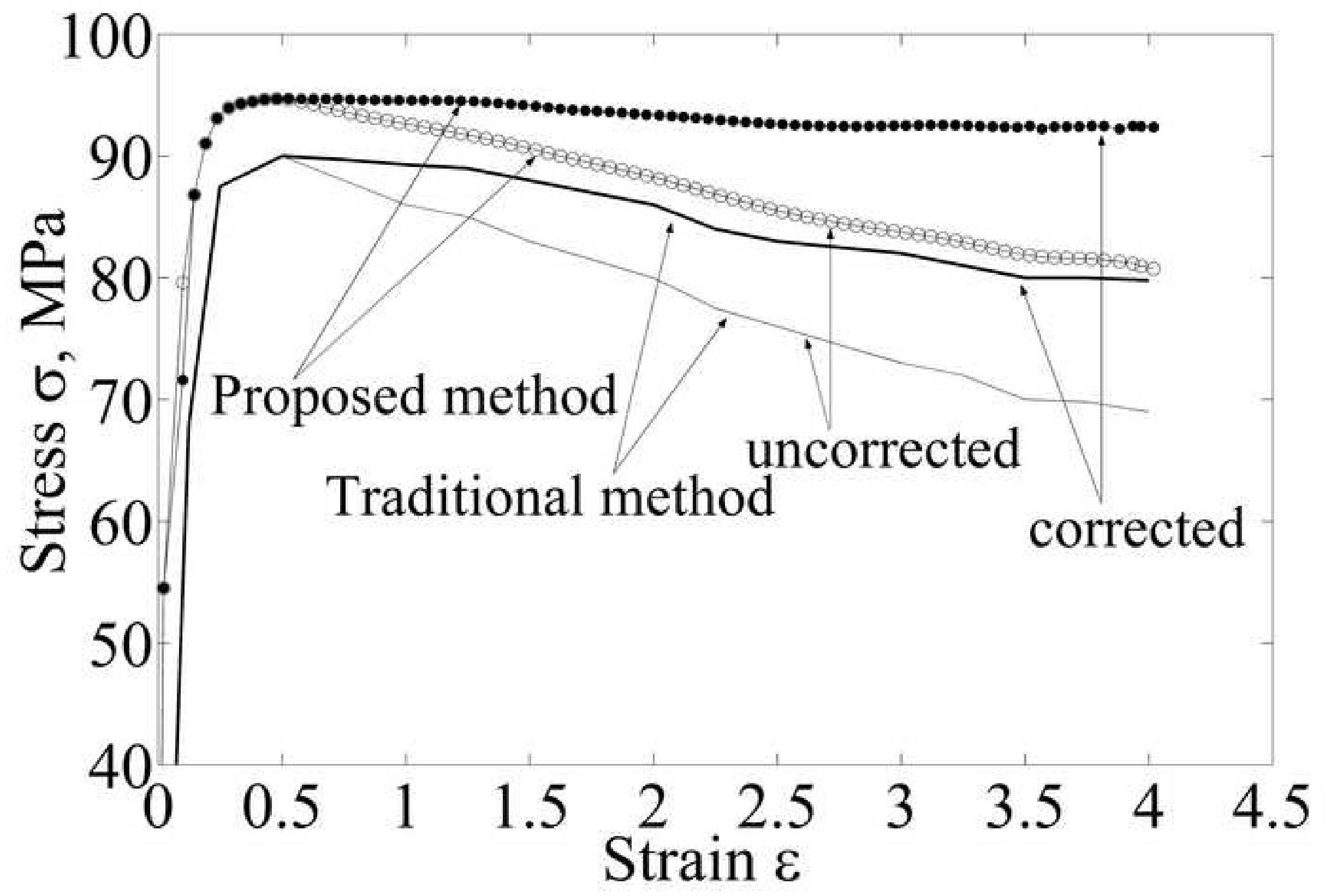



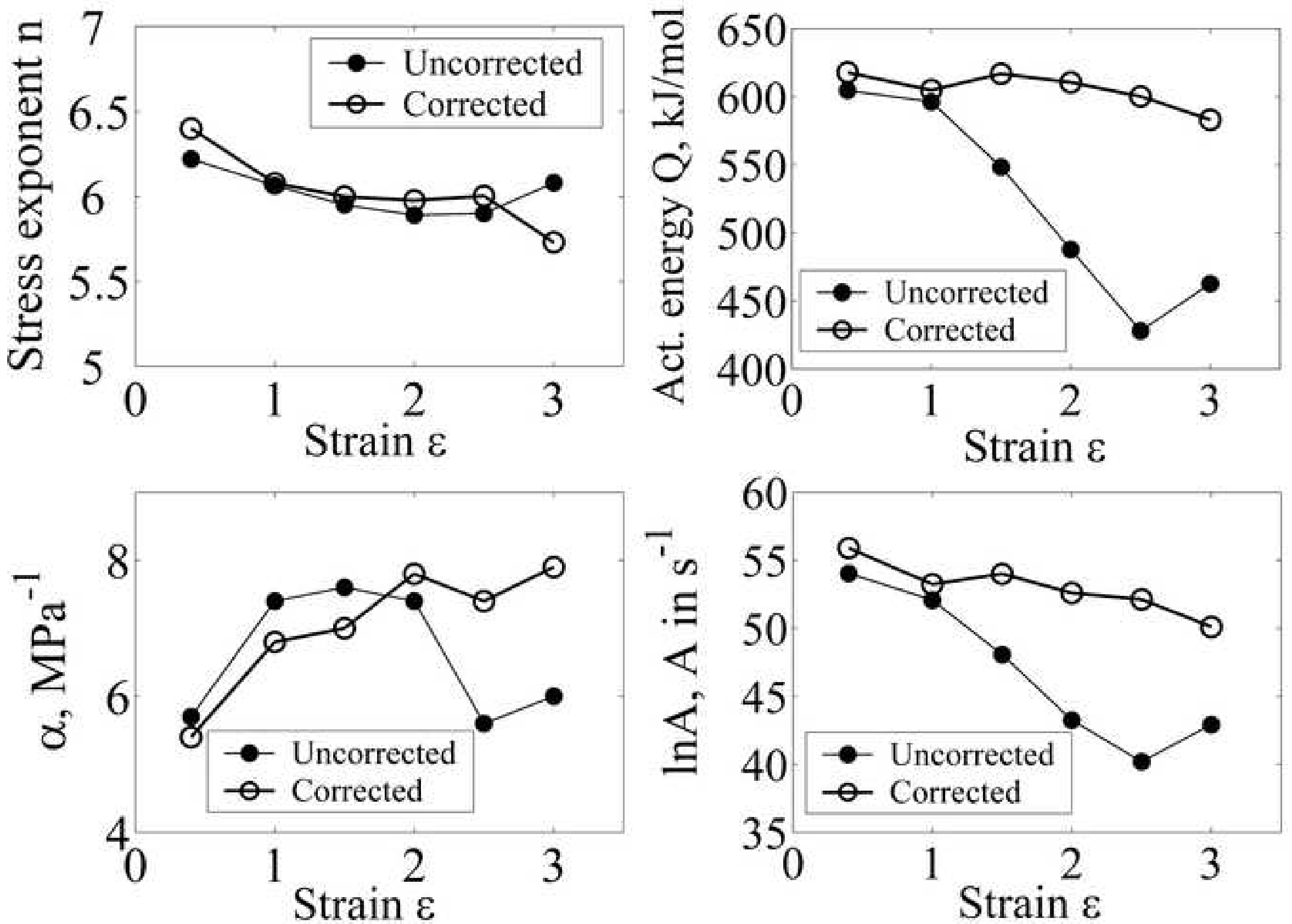
TABLES

\begin{tabular}{|c|c|cccc|}
\hline & \multirow{3}{*}{ Strain rate, $\mathrm{s}^{-1}$} & \multicolumn{4}{|c|}{ Temperature, ${ }^{\circ} \mathrm{C}$} \\
\cline { 3 - 6 } & & 975 & 1050 & 1125 & 1200 \\
\hline \multirow{4}{*}{ (a) } & 0.005 & 5.9 & 4.1 & 1.5 & 1 \\
& 0.05 & 10.4 & 10.9 & 2.8 & 1.8 \\
& 0.5 & -- & 11.1 & 7.3 & 3.1 \\
& 5 & -- & 16.9 & 6.8 & 3.7 \\
\hline & 0.005 & -- & 8.6 & 2.5 & 1.8 \\
& 0.05 & -- & 20.2 & 5.6 & 3.4 \\
(b) & 0.5 & -- & 19.7 & 10.2 & 5.1 \\
& 5 & -- & 24.5 & 12.9 & 9.2 \\
\hline
\end{tabular}

Table 1. Increment of stress, in MPa, at (a) $\varepsilon=1.5$ and b) $\varepsilon=3$ for the corrected tests for all initial temperatures and strain rates.

\begin{tabular}{|c|c|c|c|c|c|c|}
\hline Strain & $\mathrm{n}$ & $\mathrm{Q}, \mathrm{kJ} / \mathrm{mol}$ & $\alpha, \mathrm{MPa}^{-1}$ & $\ln \mathrm{A}, \mathrm{A}$ in s & $\mathrm{R}^{2}$ & $\mathrm{~F}$ \\
\hline 0.5 & 6.43 & 622.01 & 0.0055 & 55.74 & 0.987 & 441.84 \\
\hline 1 & 6.09 & 609.07 & 0.0068 & 53.19 & 0.984 & 345.77 \\
\hline 1.5 & 6.03 & 620.89 & 0.007 & 54.26 & 0.979 & 216.95 \\
\hline 2 & 5.97 & 610.77 & 0.0077 & 52.65 & 0.982 & 241.35 \\
\hline 2.5 & 6.00 & 600.37 & 0.0074 & 52.12 & 0.981 & 228.59 \\
\hline 3 & 5.73 & 583.24 & 0.0079 & 50.11 & 0.982 & 244.91 \\
\hline
\end{tabular}

Table 2. Garofalo equation parameters as a function of strain. The goodness of the fit is given by the parameters $\mathrm{R}^{2}$ and $\mathrm{F}$ of Fisher-Snedecor. 